\title{
A Time-Critical Investigation of Parameter Tuning in Differential Evolution for Non-Linear Global Optimization
}

\author{
Jia Hui Ong ${ }^{\# 1}$, Jason Teo ${ }^{\# 2}$ \\ \# Evolutionary Computing Lab, Faculty of Computing and Informatics, Universiti Malaysia Sabah, Sabah, Malaysia



\begin{abstract}
Parameter searching is one of the most important aspects in getting favorable results in optimization problems. It is even more important if the optimization problems are limited by time constraints. In a limited time constraint problems, it is crucial for any algorithms to get the best results or near-optimum results. In a previous study, Differential Evolution (DE) has been found as one of the best performing algorithms under time constraints. As this has help in answering which algorithm that yields results that are near-optimum under a limited time constraint. Hence to further enhance the performance of DE under time constraint evaluation, a throughout parameter searching for population size, mutation constant and $f$ constant have been carried out. CEC 2015 Global Optimization Competition's 15 scalable test problems are used as test suite for this study. In the previous study the same test suits has been used and the results from DE will be use as the benchmark for this study since it shows the best results among the previous tested algorithms. Eight different populations size are used and they are 10, 30, 50, 100, 150, 200, 300, and 500. Each of these populations size will run with mutation constant of 0.1 until 0.9 and from 0.1 until 0.9 . It was found that population size $100, \mathrm{Cr}=0.9$, $\mathrm{F}=\mathbf{0 . 5}$ outperform the benchmark results. It is also observed from the results that good higher $\mathrm{Cr}$ around 0.8 and 0.9 with low $\mathrm{F}$ around 0.3 to 0.4 yields good results for DE under time constraints evaluation
\end{abstract}

Keywords - evolutionary optimization; time-limited optimization; DE; expensive optimization problems; parameter searching

\section{INTRODUCTION}

Optimization problems are mostly evaluated by using number of evaluation budget. Within the given number of evaluations, an algorithm has to solve the optimizations problems without taking the amount of time used into consideration. Top conferences such as GECCO and CEC were among the platform use by researcher to show their works done on solving and finding the best solutions for the given test problems or on a particular optimization problems. CEC test suites only focus on finding best solutions without taking time consideration into account. CEC 2014 introduce a competition of real-parameter single objective expensive optimization that focus on achieving the optimum solution although it was called an expensive optimization competition, their focus was on the solutions provided by the algorithms with more dimensions to be solved. The organizers also allows participant to implement surrogatesmodel to aid their algorithms. Some of the works exhibited in GECCO 2010 are Zhou and Tan [1] who presented their work on PSO with triggered mutation, Chen [2] presented PSO with self-adjusting neighbors. Hildebrandt [3] presented the usage of GP in solving the complex shop floor scenarios. Similar to CEC conferences, the main focus of the papers presented is to solve optimization problems by providing the best solutions no matter how much time is taken.

Estimation or approximating the fitness is one of the method used by researcher to try and solved the problem face in expensive optimization problems. Instance-based learning method, machine learning method and statistical learning method are three popular method used in fitness approximation. Instance-based method entails transforming the original functions to linear ones, and then using a linear programming technique, such as the Frank-Wolfe method [4] or Powell's quadratic approximation [5]. In machine learning, the techniques available are Clustering, Multilayer Perception Neural Networks and decision tree. Statistical Learning methods for fitness approximation (basically statistical learning models) as applied to EAs have gained much interest among researchers, and have been used in several successful GA packages. In these methods, single or multiple models are built during the optimization process to approximate the original fitness function. These models are also referred to as approximate models, surrogates or metamodels. Among these models, Polynomial Models, Kriging Models, and Support Vector Machines (SVM) are the most 
commonly used. Although fitness approximation were able to decrease the time of convergence, the question of which algorithms performs the best is a given critical time frame left unanswered. Likewise the focus of fitness approximation is to achieve best solution faster.

Researches that focus on stopping criteria [6], [7], [8] focus on how to stop the optimization process when the solutions reached optimum results. Conventional optimization process use number of evaluations as the termination criteria but it is not practical as the concern of these researches is to save cost and time in real world and expensive optimization problems. Some of the suggestion mentions in these researches are to compare other algorithms with the stopping criteria mention. But still the question of how and what is the performance of PSO, DE and SEA algorithms in a given time frame optimizations problems are not answer.

In expensive optimization problems, researcher address the problems of limited resources and time in running the large number of evaluations in order to obtain the best solutions. Chen [9] used PSO aided MIMO in transceiver design in order to obtain to the best solutions and at the same time lower the computational complexity and time complexity. Vasile and Croisard [10] tackle the space mission design in their work. The main focus of their work is to reduce the time take to compute the space mission design under uncertainty. Researcher work on engineering problems [11], network design [12], word analysis [13], digital circuits[14] all these real-world expensive optimization applications focus on reducing the complexity of the optimizations process. It can be observed that reducing time taken to obtain best solution were the focus of these researchers. This shows how important time is in real world applications. It is crucial to obtain solutions as fast as possible where expensive resources are involved. Yet if the questions of which algorithms that can produce ideal solutions in a given short time frame cannot be answer even though it is observe that time plays an important aspect in real-world optimization problems.

\section{METHOD}

In CEC 2015, a competition on expensive optimization problems were organized. The benchmark problems used in the competition are used in this study. It comprises from $f 1$ to $f 15$ benchmark optimization problems as shown in Table I.

In Table II, the results for the previous study [15] are shown. These results are used as benchmark in the following results. The settings for the previous $\mathrm{DE}$ are as follow:

- $\quad$ population size $100, \mathrm{Cr}=.9, \mathrm{~F}=.2$,

The benchmark results allow us to have a measurement of how each parameter setting is performing.

TABLE I.

SUMMARY OF CEC 2015 EXPENSIVE OPTIMIZATION PROBLEMS

\begin{tabular}{|l|l|c|}
\hline No. & \multicolumn{1}{|c|}{ Function } & Fi $^{*}$ \\
\hline 1 & Rotated Bent Cigar Function & 100 \\
\hline 2 & Rotated Discus Function & 200 \\
\hline 3 & Shifted and Rotated Weierstrass Function & 300 \\
\hline 4 & Shifted and Rotated Schwefel's Function & 400 \\
\hline 5 & Shifted and Rotated Katsuura Function & 500 \\
\hline 6 & Shifted and Rotated HappyCat Function & 600 \\
\hline
\end{tabular}

\begin{tabular}{|l|l|l|}
\hline 7 & Shifted and Rotated HGBat Function & 700 \\
\hline 8 & $\begin{array}{l}\text { Shifted and Rotated Expanded Griewank's } \\
\text { plus Rosenbrock's Function }\end{array}$ & 800 \\
\hline 9 & $\begin{array}{l}\text { Shifted and Rotated Expanded Scaffer's F6 } \\
\text { Function }\end{array}$ & 900 \\
\hline 10 & Hybrid Function 1 (N=3) & 1000 \\
\hline 11 & Hybrid Function 2 $(\mathrm{N}=4)$ & 1100 \\
\hline 12 & Hybrid Function 3 $(\mathrm{N}=5)$ & 1200 \\
\hline 13 & Composition Function 1 $(\mathrm{N}=5)$ & 1300 \\
\hline 14 & Composition Function 2 $(\mathrm{N}=3)$ & 1400 \\
\hline 15 & Composition Function 3 $(\mathrm{N}=5)$ & 1500 \\
\hline
\end{tabular}

TABLE II.

DE RESULTS FOR PREVIOUS STUDY

\begin{tabular}{|c|c|}
\hline $\mathbf{F}$ & DE \\
\hline 1 & $8.68 \mathrm{E}-05$ \\
\hline 2 & $0.00 \mathrm{E}+00$ \\
\hline 3 & $1.10 \mathrm{E}+01$ \\
\hline 4 & $9.32 \mathrm{E}+02$ \\
\hline 5 & $1.83 \mathrm{E}+00$ \\
\hline 6 & $9.65 \mathrm{E}-02$ \\
\hline 7 & $8.01 \mathrm{E}-02$ \\
\hline 8 & $1.56 \mathrm{E}+00$ \\
\hline 9 & $3.01 \mathrm{E}+00$ \\
\hline 10 & $1.58 \mathrm{E}+01$ \\
\hline 11 & $5.95 \mathrm{E}+00$ \\
\hline 12 & $4.88 \mathrm{E}+01$ \\
\hline 13 & $3.16 \mathrm{E}+02$ \\
\hline 14 & $1.98 \mathrm{E}+02$ \\
\hline 15 & $4.87 \mathrm{E}+02$ \\
\hline
\end{tabular}

\section{EXPERIMENT SETUP}

For this experiment a time threshold is set to 300 milliseconds and once this threshold is reached the algorithm have to stop immediately and the best solution up to that moment are saved. The number of evaluations done in 300 milliseconds was recorded as well, in order to know how many evaluations can be done using different parameter under 300 milliseconds. There will be eight different population size and they are 10, 30, 50, 100, 150, 200, 300 and 500. Each of these population sizes will be run using different mutation and $f$ constants. The mutation and $f$ constants are as follow:

- $\mathrm{Cr}=0.1,0.2,0.3,0.4,0.5,0.6,0.7,0.8$ and 0.9

- $\quad F=0.1,0.2,0.3,0.4,0.5,0.6,0.7,0.8$ and 0.9

\section{IV.EXPERIMENT RESULTS}

Due to the large amount of tables results obtained, only the best set of parameter from each population are shown in the Appendix section. In population size of 10, all the results obtained performs much worse that the benchmark results. Form all of the parameter, $\mathrm{Cr}=0.5 \mathrm{~F}=0.7$ performs the best within population size of 10 . Hence in Table III, the overall results for $\mathrm{Cr}=0.5$ are shown and the best $\mathrm{Cr}=0.5 \mathrm{~F}=0.7$ are shown in Table IV together with the percentage of change from the benchmark results. Since the test suite is a minimization problems, negative percentage highlighted in red shows that the current results are actually performing better than the benchmark results. The overall change shown in Table IV indicates the overall changes of the fitness against the benchmark results. $\mathrm{Cr}=0.5 \mathrm{~F}=0.7$ overall changes is $561.23 \%$, although it is very high but this figure is the lowest in population size 10 . In population size of 30 , 
$\mathrm{Cr}=0.8 \mathrm{~F}=0.6$ performs the best within population size of 30 . Hence in Table $\mathrm{V}$, the overall results for $\mathrm{Cr}=0.8$ are shown and the best $\mathrm{Cr}=0.8 \mathrm{~F}=0.6$ are shown in Table VI together with the percentage of change from the benchmark results. $\mathrm{Cr}=0.5 \mathrm{~F}=0.7$ overall changes is $-51.47 \%$,

In population size of $50, \mathrm{Cr}=0.9 \mathrm{~F}=0.6$ performs the best within population size of 50. Hence in Table VII, the overall results for $\mathrm{Cr}=0.9$ are shown and the best $\mathrm{Cr}=0.9 \mathrm{~F}=0.6$ are shown in Table VIII together with the percentage of change from the benchmark results. $\mathrm{Cr}=0.5 \mathrm{~F}=0.7$ overall changes is $-58.86 \%$, only $f$ number 6,7 and 10 results is worse than the benchmark results while other $f$ number performs better in $\mathrm{Cr}=0.5 \mathrm{~F}=0.7$. In population size of $100, \mathrm{Cr}=0.8 \mathrm{~F}=0.4$ performs the best within population size of 100 . Hence in Table IX, the overall results for $\mathrm{Cr}=0.8$ are shown and the best $\mathrm{Cr}=0.8 \mathrm{~F}=0.4$ are shown in Table $\mathrm{X}$ together with the percentage of change from the benchmark results. $\mathrm{Cr}=0.8$ $\mathrm{F}=0.4$ overall changes is $-34.73 \%$, only $f$ number 1,2 and 10 results are worse than the benchmark results while other $f$ number performs better in $\mathrm{Cr}=0.8 \mathrm{~F}=0.4$. For population size of $150, \mathrm{Cr}=0.8 \mathrm{~F}=0.4$ performs the best within population size of 150. Hence in Table XI, the overall results for $\mathrm{Cr}=0.8$ are shown and the best $\mathrm{Cr}=0.8 \mathrm{~F}=0.4$ are shown in Table XII together with the percentage of change from the benchmark results. $\mathrm{Cr}=0.8 \mathrm{~F}=0.4$ overall changes is $-35.18 \%$ and only $f$ number $1,2,10$ and 12 results are worse than the benchmark results while other $f$ number performs better in $\mathrm{Cr}=0.8 \mathrm{~F}=0.4$.

In population size of $200, \mathrm{Cr}=0.9 \mathrm{~F}=0.4$ performs the best within population size of 200. Hence in Table XIII, the overall results for $\mathrm{Cr}=0.9$ are shown and the best $\mathrm{Cr}=0.9$ $\mathrm{F}=0.4$ are shown in Table XIV together with the percentage of change from the benchmark results. $\mathrm{Cr}=0.9 \mathrm{~F}=0.4$ overall changes is $-30.28 \%$.

While population size of $300, \mathrm{Cr}=0.9 \mathrm{~F}=0.3$ performs the best within population size of 300 . Hence in Table XV, the overall results for $\mathrm{Cr}=0.9$ are shown and the best $\mathrm{Cr}=0.9$ $\mathrm{F}=0.3$ are shown in Table XVI together with the percentage of change from the benchmark results. $\mathrm{Cr}=0.9 \mathrm{~F}=0.3$ overall changes is $-29.69 \%$. In population size of $500, \mathrm{Cr}=0.9 \mathrm{~F}=0.3$ performs the best within population size of 500 . Hence in Table XVII, the overall results for $\mathrm{Cr}=0.9$ are shown and the best $\mathrm{Cr}=0.9 \mathrm{~F}=0.3$ are shown in Table XVIII together with the percentage of change from the benchmark results. $\mathrm{Cr}=0.9$ $\mathrm{F}=0.3$ overall changes is $-32.08 \%$.

From all the results obtained, population size $50 \mathrm{Cr}=0.5$ $\mathrm{F}=0.7$ has the best overall changes which is $-58.86 \%$ but it did not perform better for function number 6,7 and 10 . While in Table XIX population size $100, \mathrm{Cr}=0.9, \mathrm{~F}=0.5$, manage to outperform benchmark results in all of the functions although with only overall changes of $-32.42 \%$. Hence it can concluded that population size $100, \mathrm{Cr}=0.9, \mathrm{~F}=0.5$ is the ideal parameter settings for DE under time evaluation constraints.

\section{CONCLUSIONS AND FUTURE WORKS}

It is observed that with high $\mathrm{Cr}$ and lower $\mathrm{F}$, it can yield better results for DE under time constraints evaluations. The sweet spots for $\mathrm{Cr}$ are 0.8 and 0.9 and $\mathrm{F}$ is 0.3 to 0.5 . From the results obtain it is observed that population size 50, 100 and 150 yields much better results that the other population size. For future works, different variant of DE should be study under time constraint evaluations. In hopes that with different variant of DE, the performance and results obtained under time constraint evaluations can improve to near optimum solutions.

\section{REFERENCES}

[1] Y.Zhou, and Y. Tan.:Particle Swarm Optimization with triggered mutation and its implementation based on gpu. In Proceedings of Genetic and Evolutionary Computation Conference ,GECCOJuly 07-11, 2010, Portland, Oregon, USA (2010).

[2] Z. Chen , Z. He , C. Zhang.:Particle swarm optimizer with selfadjusting neighborhoods,. In In Proceedings of Genetic and Evolutionary Computation Conference ,GECCOJuly 07-11, 2010, Portland, Oregon, USA (2010).

[3] T. Hildebrandt , J. Heger , B.Scholz-Reiter.: Towards improved dispatching rules for complex shop floor scenarios: a genetic programming approach, Proceedings of the 12th annual conference on Genetic and evolutionary computation, July 07-11, 2010, Portland, Oregon, USA (2010)

[4] Frank, M.; Wolfe, P.: An algorithm for quadratic programming. Naval Research Logistics Quarterly 3: 95 (1956).

[5] Powell M.J.D.: UOBYQA: unconstrained optimization by quadratic approximation. Math. Program. 92, 555-582. (2002).

[6] M. Iwan, R. Akmeliawati, T. Faisal, H., M.A.A. AlAssadi.:Performance Comparison of Differential Evolution and Particle Swarm Optimization in Constrained Optimization.International Symposium on Robotics and Intelligent Sensors (2012).

[7] K. Zielinski, R. Laur.: Stopping Criteria for a Constrained SingleObjective Particle Swarm Optimization Algorithm Informatica 31(1):51-59 - March (2007)

[8] A. Chaudhuri, R. T. Haftka.: A Stopping Criterion for Surrogate Based Optimization using EGO, 10th World Congress on Structural and Multidisciplinary Optimization, Orlando, FL, USA, May 20-24, (2013)

[9] S. Chen, W. Yao, H. R. Palally, L. Hanzo.:Particle Swarm Optimisation Aided MIMO Transceiver Designs. Computational Intelligence in Expensive Optimization Problems Volume 2 of Adaptation Learning and Optimization pp 487-511 (2010).

[10] M. Vasile, N. Croisard.:Robust Preliminary Space Mission Design Under Uncertainty.Computational Intelligence in Expensive Optimization Problems. Vol. 2. p. 543-570 Adaptation Learning and Optimization; Vol. 2.Berlin, (2010).

[11] S. Kumar ,\& K.F. Bauer. Exploring the use of Lean Thinking and Six Sigma in public housing authorities. The Quality Management Journal, vol. 17, no. 1, p. 29-46. (2010).

[12] J. Mattingley, and S. Boyd.: Real Time Convex Optimization in Signal Processing. IEEE Signal Processing Magazine. (2010).

[13] W. M. Yamada, T. W. Berger.: Isolated Word Analysis Using Biologically-Based Neural Networks. Computational Intelligence in Expensive Optimization ProblemsVolume 2 of the Adaptation Learning and Optimization pp 637-670 (2010).

[14] C. Pilato, D. Loiacono, A. Tumeo, F. Ferrandi, P. L. Lanzi, D. Sciuto.: Speeding-Up Expensive Evaluations in High-Level Synthesis Using Solution modelling and Fitness In heritance. Computational Intelligence in Expensive Optimization Problems. (2010).

[15] J. H. Ong, J. Teo.: A Comprehensive Comparison of Evolutionary Optimization Limited by Number of Evaluations against Time Constrained. ARPN Journal of Engineering and Applied Science (JEAS). (under review) 


\section{APPENDIX}

TABLE III.

OVERALL RESULTS FOR POPULATION SIZE $10, \mathrm{CR}=0.5$

\begin{tabular}{|r|c|c|c|c|c|c|c|c|c|}
\hline $\begin{array}{r}\mathbf{F} / \\
\boldsymbol{f}\end{array}$ & $\mathbf{0 . 1}$ & $\mathbf{0 . 2}$ & $\mathbf{0 . 3}$ & $\mathbf{0 . 4}$ & $\mathbf{0 . 5}$ & $\mathbf{0 . 6}$ & $\mathbf{0 . 7}$ & $\mathbf{0 . 8}$ & $\mathbf{0 . 9}$ \\
\hline 1 & $2.52 \mathrm{E}+09$ & $1.02 \mathrm{E}+09$ & $2.89 \mathrm{E}+08$ & $1.18 \mathrm{E}+08$ & $2.22 \mathrm{E}+07$ & $1.99 \mathrm{E}+05$ & $9.22 \mathrm{E}+03$ & $9.01 \mathrm{E}+03$ & $1.98 \mathrm{E}+04$ \\
\hline 2 & $2.36 \mathrm{E}+04$ & $1.65 \mathrm{E}+04$ & $1.09 \mathrm{E}+04$ & $7.64 \mathrm{E}+03$ & $2.68 \mathrm{E}+03$ & $2.69 \mathrm{E}+01$ & $4.33 \mathrm{E}-01$ & $1.90 \mathrm{E}+01$ & $1.39 \mathrm{E}+02$ \\
\hline 3 & $7.31 \mathrm{E}+00$ & $5.90 \mathrm{E}+00$ & $6.14 \mathrm{E}+00$ & $7.41 \mathrm{E}+00$ & $9.46 \mathrm{E}+00$ & $1.08 \mathrm{E}+01$ & $1.15 \mathrm{E}+01$ & $1.12 \mathrm{E}+01$ & $1.15 \mathrm{E}+01$ \\
\hline 4 & $3.37 \mathrm{E}+02$ & $2.04 \mathrm{E}+02$ & $9.43 \mathrm{E}+01$ & $8.38 \mathrm{E}+01$ & $4.89 \mathrm{E}+01$ & $6.33 \mathrm{E}+01$ & $5.67 \mathrm{E}+01$ & $4.28 \mathrm{E}+01$ & $4.45 \mathrm{E}+01$ \\
\hline 5 & $1.95 \mathrm{E}+00$ & $1.90 \mathrm{E}+00$ & $1.90 \mathrm{E}+00$ & $1.99 \mathrm{E}+00$ & $2.00 \mathrm{E}+00$ & $1.98 \mathrm{E}+00$ & $1.93 \mathrm{E}+00$ & $1.95 \mathrm{E}+00$ & $1.87 \mathrm{E}+00$ \\
\hline 6 & $2.39 \mathrm{E}+00$ & $1.05 \mathrm{E}+00$ & $6.08 \mathrm{E}-01$ & $2.69 \mathrm{E}-01$ & $1.36 \mathrm{E}-01$ & $9.66 \mathrm{E}-02$ & $1.09 \mathrm{E}-01$ & $1.28 \mathrm{E}-01$ & $1.50 \mathrm{E}-01$ \\
\hline 7 & $2.21 \mathrm{E}+01$ & $9.00 \mathrm{E}+00$ & $5.30 \mathrm{E}+00$ & $8.97 \mathrm{E}-01$ & $1.35 \mathrm{E}-01$ & $9.48 \mathrm{E}-02$ & $6.50 \mathrm{E}-02$ & $6.76 \mathrm{E}-02$ & $7.61 \mathrm{E}-02$ \\
\hline 8 & $2.16 \mathrm{E}+03$ & $4.85 \mathrm{E}+02$ & $5.57 \mathrm{E}+01$ & $1.58 \mathrm{E}+00$ & $1.31 \mathrm{E}+00$ & $1.41 \mathrm{E}+00$ & $1.65 \mathrm{E}+00$ & $1.78 \mathrm{E}+00$ & $1.85 \mathrm{E}+00$ \\
\hline 9 & $3.02 \mathrm{E}+00$ & $2.70 \mathrm{E}+00$ & $2.10 \mathrm{E}+00$ & $2.15 \mathrm{E}+00$ & $2.26 \mathrm{E}+00$ & $2.83 \mathrm{E}+00$ & $3.20 \mathrm{E}+00$ & $3.49 \mathrm{E}+00$ & $3.72 \mathrm{E}+00$ \\
\hline 10 & $1.54 \mathrm{E}+05$ & $7.80 \mathrm{E}+04$ & $1.02 \mathrm{E}+04$ & $2.68 \mathrm{E}+03$ & $1.37 \mathrm{E}+03$ & $1.54 \mathrm{E}+03$ & $2.88 \mathrm{E}+03$ & $5.46 \mathrm{E}+03$ & $1.37 \mathrm{E}+04$ \\
\hline 11 & $7.58 \mathrm{E}+00$ & $7.02 \mathrm{E}+00$ & $4.59 \mathrm{E}+00$ & $4.59 \mathrm{E}+00$ & $4.75 \mathrm{E}+00$ & $6.54 \mathrm{E}+00$ & $7.77 \mathrm{E}+00$ & $9.71 \mathrm{E}+00$ & $1.29 \mathrm{E}+01$ \\
\hline 12 & $1.21 \mathrm{E}+02$ & $9.34 \mathrm{E}+01$ & $7.28 \mathrm{E}+01$ & $6.71 \mathrm{E}+01$ & $7.02 \mathrm{E}+01$ & $7.73 \mathrm{E}+01$ & $1.10 \mathrm{E}+02$ & $1.73 \mathrm{E}+02$ & $2.06 \mathrm{E}+02$ \\
\hline 13 & $3.78 \mathrm{E}+02$ & $3.38 \mathrm{E}+02$ & $3.29 \mathrm{E}+02$ & $3.19 \mathrm{E}+02$ & $3.16 \mathrm{E}+02$ & $3.15 \mathrm{E}+02$ & $3.19 \mathrm{E}+02$ & $3.26 \mathrm{E}+02$ & $3.26 \mathrm{E}+02$ \\
\hline 14 & $2.05 \mathrm{E}+02$ & $2.00 \mathrm{E}+02$ & $1.98 \mathrm{E}+02$ & $1.98 \mathrm{E}+02$ & $1.98 \mathrm{E}+02$ & $1.99 \mathrm{E}+02$ & $2.01 \mathrm{E}+02$ & $2.01 \mathrm{E}+02$ & $2.01 \mathrm{E}+02$ \\
\hline 15 & $4.60 \mathrm{E}+02$ & $4.23 \mathrm{E}+02$ & $3.99 \mathrm{E}+02$ & $4.44 \mathrm{E}+02$ & $4.78 \mathrm{E}+02$ & $5.66 \mathrm{E}+02$ & $5.66 \mathrm{E}+02$ & $5.85 \mathrm{E}+02$ & $5.91 \mathrm{E}+02$ \\
\hline
\end{tabular}

TABLE IV.

RESULTS FOR POPULATION SIZE $10, \mathrm{CR}=0.5,0.7$

\begin{tabular}{|c|c|c|}
\hline $\boldsymbol{f}$ & Fitness & Percentage of Change \\
\hline 1 & $9.22 \mathrm{E}+03$ & $>1000.00 \%$ \\
\hline 2 & $4.33 \mathrm{E}-01$ & $43.33 \%$ \\
\hline 3 & $1.15 \mathrm{E}+01$ & $3.84 \%$ \\
\hline 4 & $5.67 \mathrm{E}+01$ & $-93.91 \%$ \\
\hline 5 & $1.93 \mathrm{E}+00$ & $5.34 \%$ \\
\hline 6 & $1.09 \mathrm{E}-01$ & $13.34 \%$ \\
\hline 7 & $6.50 \mathrm{E}-02$ & $-18.80 \%$ \\
\hline 8 & $1.65 \mathrm{E}+00$ & $5.38 \%$ \\
\hline 9 & $3.20 \mathrm{E}+00$ & $6.52 \%$ \\
\hline 10 & $2.88 \mathrm{E}+03$ & $>1000.00 \% \%$ \\
\hline 11 & $7.77 \mathrm{E}+00$ & $30.73 \%$ \\
\hline 12 & $1.10 \mathrm{E}+02$ & $124.94 \%$ \\
\hline 13 & $3.19 \mathrm{E}+02$ & $0.94 \%$ \\
\hline 14 & $2.01 \mathrm{E}+02$ & $1.15 \%$ \\
\hline 15 & $5.66 \mathrm{E}+02$ & $16.10 \%$ \\
\hline & Overall & $561.23 \%$ \\
\hline & & \\
\hline
\end{tabular}


TABLE V.

OVERALL RESULTS FOR POPULATION SIZE $30 \mathrm{CR}=0.8$

\begin{tabular}{|r|c|c|c|c|c|c|c|c|c|}
\hline $\begin{array}{r}\text { FR/ } \\
\boldsymbol{f}\end{array}$ & $\mathbf{0 . 1}$ & $\mathbf{0 . 2}$ & $\mathbf{0 . 3}$ & $\mathbf{0 . 4}$ & $\mathbf{0 . 5}$ & $\mathbf{0 . 6}$ & $\mathbf{0 . 7}$ & $\mathbf{0 . 8}$ & $\mathbf{0 . 9}$ \\
\hline 1 & $2.74 \mathrm{E}+08$ & $3.34 \mathrm{E}+07$ & $1.00 \mathrm{E}+06$ & $9.56 \mathrm{E}+03$ & $8.53 \mathrm{E}+03$ & $2.81 \mathrm{E}+01$ & $1.47 \mathrm{E}-05$ & $1.56 \mathrm{E}-01$ & $2.35 \mathrm{E}+02$ \\
\hline 2 & $1.62 \mathrm{E}+04$ & $9.06 \mathrm{E}+03$ & $6.34 \mathrm{E}+03$ & $2.65 \mathrm{E}+03$ & $4.61 \mathrm{E}+00$ & $6.48 \mathrm{E}-09$ & $6.02 \mathrm{E}-09$ & $5.56 \mathrm{E}-09$ & $5.60 \mathrm{E}-09$ \\
\hline 3 & $4.87 \mathrm{E}+00$ & $4.68 \mathrm{E}+00$ & $5.47 \mathrm{E}+00$ & $6.49 \mathrm{E}+00$ & $7.25 \mathrm{E}+00$ & $7.67 \mathrm{E}+00$ & $7.71 \mathrm{E}+00$ & $7.69 \mathrm{E}+00$ & $7.81 \mathrm{E}+00$ \\
\hline 4 & $5.04 \mathrm{E}+01$ & $1.87 \mathrm{E}+01$ & $9.05 \mathrm{E}+00$ & $1.34 \mathrm{E}+01$ & $2.10 \mathrm{E}+01$ & $5.23 \mathrm{E}+01$ & $1.02 \mathrm{E}+02$ & $5.67 \mathrm{E}+01$ & $1.39 \mathrm{E}+02$ \\
\hline 5 & $1.30 \mathrm{E}+00$ & $1.32 \mathrm{E}+00$ & $1.31 \mathrm{E}+00$ & $1.28 \mathrm{E}+00$ & $1.24 \mathrm{E}+00$ & $1.27 \mathrm{E}+00$ & $1.22 \mathrm{E}+00$ & $1.26 \mathrm{E}+00$ & $1.27 \mathrm{E}+00$ \\
\hline 6 & $7.79 \mathrm{E}-01$ & $1.58 \mathrm{E}-01$ & $6.48 \mathrm{E}-02$ & $5.56 \mathrm{E}-02$ & $6.64 \mathrm{E}-02$ & $7.49 \mathrm{E}-02$ & $8.95 \mathrm{E}-02$ & $9.87 \mathrm{E}-02$ & $1.20 \mathrm{E}-01$ \\
\hline 7 & $3.48 \mathrm{E}+00$ & $4.66 \mathrm{E}-01$ & $1.97 \mathrm{E}-01$ & $1.05 \mathrm{E}-01$ & $7.29 \mathrm{E}-02$ & $8.14 \mathrm{E}-02$ & $7.29 \mathrm{E}-02$ & $7.03 \mathrm{E}-02$ & $6.91 \mathrm{E}-02$ \\
\hline 8 & $1.25 \mathrm{E}+01$ & $1.03 \mathrm{E}+00$ & $6.80 \mathrm{E}-01$ & $6.02 \mathrm{E}-01$ & $8.25 \mathrm{E}-01$ & $1.09 \mathrm{E}+00$ & $1.14 \mathrm{E}+00$ & $1.23 \mathrm{E}+00$ & $1.27 \mathrm{E}+00$ \\
\hline 9 & $1.86 \mathrm{E}+00$ & $1.52 \mathrm{E}+00$ & $1.15 \mathrm{E}+00$ & $1.25 \mathrm{E}+00$ & $1.61 \mathrm{E}+00$ & $2.17 \mathrm{E}+00$ & $2.32 \mathrm{E}+00$ & $2.22 \mathrm{E}+00$ & $2.31 \mathrm{E}+00$ \\
\hline 10 & $6.81 \mathrm{E}+04$ & $1.52 \mathrm{E}+04$ & $1.22 \mathrm{E}+03$ & $1.47 \mathrm{E}+03$ & $1.64 \mathrm{E}+02$ & $1.33 \mathrm{E}+02$ & $1.25 \mathrm{E}+02$ & $1.54 \mathrm{E}+02$ & $2.18 \mathrm{E}+02$ \\
\hline 11 & $3.38 \mathrm{E}+00$ & $2.61 \mathrm{E}+00$ & $2.23 \mathrm{E}+00$ & $2.15 \mathrm{E}+00$ & $2.81 \mathrm{E}+00$ & $3.81 \mathrm{E}+00$ & $5.06 \mathrm{E}+00$ & $6.20 \mathrm{E}+00$ & $8.30 \mathrm{E}+00$ \\
\hline 12 & $5.52 \mathrm{E}+01$ & $2.99 \mathrm{E}+01$ & $2.49 \mathrm{E}+01$ & $1.87 \mathrm{E}+01$ & $1.84 \mathrm{E}+01$ & $3.41 \mathrm{E}+01$ & $4.27 \mathrm{E}+01$ & $7.88 \mathrm{E}+01$ & $1.01 \mathrm{E}+02$ \\
\hline 13 & $2.22 \mathrm{E}+02$ & $2.14 \mathrm{E}+02$ & $2.11 \mathrm{E}+02$ & $2.10 \mathrm{E}+02$ & $2.10 \mathrm{E}+02$ & $2.15 \mathrm{E}+02$ & $2.14 \mathrm{E}+02$ & $2.10 \mathrm{E}+02$ & $2.11 \mathrm{E}+02$ \\
\hline 14 & $1.35 \mathrm{E}+02$ & $1.31 \mathrm{E}+02$ & $1.31 \mathrm{E}+02$ & $1.33 \mathrm{E}+02$ & $1.33 \mathrm{E}+02$ & $1.33 \mathrm{E}+02$ & $1.33 \mathrm{E}+02$ & $1.33 \mathrm{E}+02$ & $1.33 \mathrm{E}+02$ \\
\hline 15 & $2.17 \mathrm{E}+02$ & $2.45 \mathrm{E}+02$ & $2.35 \mathrm{E}+02$ & $2.99 \mathrm{E}+02$ & $3.55 \mathrm{E}+02$ & $3.69 \mathrm{E}+02$ & $3.79 \mathrm{E}+02$ & $3.90 \mathrm{E}+02$ & $3.96 \mathrm{E}+02$ \\
\hline
\end{tabular}

TABLE VI.

RESULTS FOR POPULATION SIZE $30, \mathrm{CR}=0.8, \mathrm{~F}=0.6$

\begin{tabular}{|c|c|c|}
\hline $\boldsymbol{f}$ & Fitness & Percentage of Change \\
\hline 1 & $2.81 \mathrm{E}+01$ & $>1000.00 \% \%$ \\
\hline 2 & $6.48 \mathrm{E}-09$ & $0.00 \%$ \\
\hline 3 & $7.67 \mathrm{E}+00$ & $-30.54 \%$ \\
\hline 4 & $5.23 \mathrm{E}+01$ & $-94.39 \%$ \\
\hline 5 & $1.27 \mathrm{E}+00$ & $-30.55 \%$ \\
\hline 6 & $7.49 \mathrm{E}-02$ & $-22.40 \%$ \\
\hline 7 & $8.14 \mathrm{E}-02$ & $1.68 \%$ \\
\hline 8 & $1.09 \mathrm{E}+00$ & $-30.24 \%$ \\
\hline 9 & $2.17 \mathrm{E}+00$ & $-27.67 \%$ \\
\hline 10 & $1.33 \mathrm{E}+02$ & $742.89 \%$ \\
\hline 11 & $3.81 \mathrm{E}+00$ & $-35.93 \%$ \\
\hline 12 & $3.41 \mathrm{E}+01$ & $-30.08 \%$ \\
\hline 13 & $2.15 \mathrm{E}+02$ & $-31.77 \%$ \\
\hline 14 & $1.33 \mathrm{E}+02$ & $-32.76 \%$ \\
\hline 15 & $3.69 \mathrm{E}+02$ & $-24.30 \%$ \\
\hline & Overall Changes & $-51.47 \%$ \\
\hline
\end{tabular}


TABLE VII.

OVERALL RESULTS FOR POPULATION SIZE $50 \mathrm{CR}=0.9$

\begin{tabular}{|r|c|c|c|c|c|c|c|c|c|}
\hline $\begin{array}{r}\text { FR/ } \\
\boldsymbol{f}\end{array}$ & $\mathbf{0 . 1}$ & $\mathbf{0 . 2}$ & $\mathbf{0 . 3}$ & $\mathbf{0 . 4}$ & $\mathbf{0 . 5}$ & $\mathbf{0 . 6}$ & $\mathbf{0 . 7}$ & $\mathbf{0 . 8}$ & $\mathbf{0 . 9}$ \\
\hline 1 & $2.63 \mathrm{E}+08$ & $1.58 \mathrm{E}+07$ & $9.12 \mathrm{E}+05$ & $7.96 \mathrm{E}+03$ & $9.25 \mathrm{E}+02$ & $5.30 \mathrm{E}-09$ & $5.51 \mathrm{E}-09$ & $7.77 \mathrm{E}-03$ & $4.00 \mathrm{E}+02$ \\
\hline 2 & $1.27 \mathrm{E}+04$ & $7.67 \mathrm{E}+03$ & $3.89 \mathrm{E}+03$ & $7.31 \mathrm{E}+02$ & $1.51 \mathrm{E}+00$ & $5.14 \mathrm{E}-09$ & $4.96 \mathrm{E}-09$ & $5.40 \mathrm{E}-09$ & $5.58 \mathrm{E}-09$ \\
\hline 3 & $5.32 \mathrm{E}+00$ & $5.39 \mathrm{E}+00$ & $5.98 \mathrm{E}+00$ & $6.79 \mathrm{E}+00$ & $7.22 \mathrm{E}+00$ & $7.61 \mathrm{E}+00$ & $7.64 \mathrm{E}+00$ & $7.62 \mathrm{E}+00$ & $7.83 \mathrm{E}+00$ \\
\hline 4 & $3.81 \mathrm{E}+01$ & $1.78 \mathrm{E}+01$ & $1.16 \mathrm{E}+01$ & $1.88 \mathrm{E}+01$ & $4.80 \mathrm{E}+01$ & $5.87 \mathrm{E}+01$ & $1.41 \mathrm{E}+02$ & $1.30 \mathrm{E}+02$ & $1.38 \mathrm{E}+02$ \\
\hline 5 & $1.18 \mathrm{E}+00$ & $1.26 \mathrm{E}+00$ & $1.25 \mathrm{E}+00$ & $1.19 \mathrm{E}+00$ & $1.21 \mathrm{E}+00$ & $1.28 \mathrm{E}+00$ & $1.23 \mathrm{E}+00$ & $1.24 \mathrm{E}+00$ & $1.28 \mathrm{E}+00$ \\
\hline 6 & $4.23 \mathrm{E}-01$ & $8.66 \mathrm{E}-02$ & $4.28 \mathrm{E}-02$ & $6.47 \mathrm{E}-02$ & $7.65 \mathrm{E}-02$ & $1.02 \mathrm{E}-01$ & $8.07 \mathrm{E}-02$ & $8.50 \mathrm{E}-02$ & $1.15 \mathrm{E}-01$ \\
\hline 7 & $1.71 \mathrm{E}+00$ & $2.98 \mathrm{E}-01$ & $1.65 \mathrm{E}-01$ & $1.18 \mathrm{E}-01$ & $9.88 \mathrm{E}-02$ & $8.89 \mathrm{E}-02$ & $8.15 \mathrm{E}-02$ & $9.03 \mathrm{E}-02$ & $9.37 \mathrm{E}-02$ \\
\hline 8 & $1.51 \mathrm{E}+01$ & $7.90 \mathrm{E}-01$ & $5.78 \mathrm{E}-01$ & $5.81 \mathrm{E}-01$ & $9.22 \mathrm{E}-01$ & $1.04 \mathrm{E}+00$ & $1.22 \mathrm{E}+00$ & $1.28 \mathrm{E}+00$ & $1.06 \mathrm{E}+00$ \\
\hline 9 & $1.81 \mathrm{E}+00$ & $1.48 \mathrm{E}+00$ & $1.20 \mathrm{E}+00$ & $1.44 \mathrm{E}+00$ & $1.87 \mathrm{E}+00$ & $1.91 \mathrm{E}+00$ & $2.06 \mathrm{E}+00$ & $2.31 \mathrm{E}+00$ & $2.00 \mathrm{E}+00$ \\
\hline 10 & $5.97 \mathrm{E}+04$ & $5.61 \mathrm{E}+03$ & $3.93 \mathrm{E}+02$ & $1.07 \mathrm{E}+02$ & $4.63 \mathrm{E}+01$ & $1.76 \mathrm{E}+01$ & $3.45 \mathrm{E}+01$ & $7.32 \mathrm{E}+01$ & $1.04 \mathrm{E}+02$ \\
\hline 11 & $3.89 \mathrm{E}+00$ & $2.35 \mathrm{E}+00$ & $1.89 \mathrm{E}+00$ & $2.03 \mathrm{E}+00$ & $2.68 \mathrm{E}+00$ & $3.32 \mathrm{E}+00$ & $4.22 \mathrm{E}+00$ & $5.06 \mathrm{E}+00$ & $7.04 \mathrm{E}+00$ \\
\hline 12 & $5.70 \mathrm{E}+01$ & $2.32 \mathrm{E}+01$ & $1.87 \mathrm{E}+01$ & $1.51 \mathrm{E}+01$ & $1.51 \mathrm{E}+01$ & $2.17 \mathrm{E}+01$ & $3.21 \mathrm{E}+01$ & $4.85 \mathrm{E}+01$ & $7.28 \mathrm{E}+01$ \\
\hline 13 & $2.22 \mathrm{E}+02$ & $2.13 \mathrm{E}+02$ & $2.10 \mathrm{E}+02$ & $2.10 \mathrm{E}+02$ & $2.10 \mathrm{E}+02$ & $2.10 \mathrm{E}+02$ & $2.10 \mathrm{E}+02$ & $2.10 \mathrm{E}+02$ & $2.12 \mathrm{E}+02$ \\
\hline 14 & $1.33 \mathrm{E}+02$ & $1.30 \mathrm{E}+02$ & $1.33 \mathrm{E}+02$ & $1.34 \mathrm{E}+02$ & $1.33 \mathrm{E}+02$ & $1.33 \mathrm{E}+02$ & $1.32 \mathrm{E}+02$ & $1.33 \mathrm{E}+02$ & $1.33 \mathrm{E}+02$ \\
\hline 15 & $2.64 \mathrm{E}+02$ & $2.50 \mathrm{E}+02$ & $2.59 \mathrm{E}+02$ & $3.09 \mathrm{E}+02$ & $3.43 \mathrm{E}+02$ & $3.75 \mathrm{E}+02$ & $3.73 \mathrm{E}+02$ & $3.78 \mathrm{E}+02$ & $3.85 \mathrm{E}+02$ \\
\hline
\end{tabular}

TABLE VIII.

RESULTS FOR POPULATION SIZE 50, $\mathrm{CR}=0.9, \mathrm{~F}=0.6$

\begin{tabular}{|c|c|c|}
\hline $\boldsymbol{f}$ & Fitness & Percentage of Change \\
\hline 1 & $5.30 \mathrm{E}-09$ & $-99.99 \%$ \\
\hline 2 & $5.14 \mathrm{E}-09$ & $0.00 \%$ \\
\hline 3 & $7.61 \mathrm{E}+00$ & $-31.10 \%$ \\
\hline 4 & $5.87 \mathrm{E}+01$ & $-93.70 \%$ \\
\hline 5 & $1.28 \mathrm{E}+00$ & $-29.84 \%$ \\
\hline 6 & $1.02 \mathrm{E}-01$ & $5.40 \%$ \\
\hline 7 & $8.89 \mathrm{E}-02$ & $11.05 \%$ \\
\hline 8 & $1.04 \mathrm{E}+00$ & $-33.20 \%$ \\
\hline 9 & $1.91 \mathrm{E}+00$ & $-36.51 \%$ \\
\hline 10 & $1.76 \mathrm{E}+01$ & $11.77 \%$ \\
\hline 11 & $3.32 \mathrm{E}+00$ & $-44.19 \%$ \\
\hline 12 & $2.17 \mathrm{E}+01$ & $-55.60 \%$ \\
\hline 13 & $2.10 \mathrm{E}+02$ & $-33.35 \%$ \\
\hline 14 & $1.33 \mathrm{E}+02$ & $-32.99 \%$ \\
\hline 15 & $3.75 \mathrm{E}+02$ & $-23.01 \%$ \\
\hline & Overall & $-58.86 \%$ \\
\hline & & \\
\hline
\end{tabular}


TABLE IX.

OVERALL RESULTS FOR POPULATION SIZE $100 \mathrm{CR}=0.8$

\begin{tabular}{|r|c|c|c|c|c|c|c|c|c|}
\hline \multicolumn{1}{|c|}{$\boldsymbol{f} /$} \\
$\boldsymbol{f}$ & $\mathbf{0 . 1}$ & $\mathbf{0 . 2}$ & $\mathbf{0 . 3}$ & $\mathbf{0 . 4}$ & $\mathbf{0 . 5}$ & $\mathbf{0 . 6}$ & $\mathbf{0 . 7}$ & $\mathbf{0 . 8}$ & $\mathbf{0 . 9}$ \\
\hline 1 & $8.02 \mathrm{E}+05$ & $7.39 \mathrm{E}+03$ & $4.89 \mathrm{E}+03$ & $1.50 \mathrm{E}+02$ & $2.53 \mathrm{E}+00$ & $3.34 \mathrm{E}+03$ & $2.78 \mathrm{E}+05$ & $8.55 \mathrm{E}+06$ & $6.65 \mathrm{E}+07$ \\
\hline 2 & $5.44 \mathrm{E}+03$ & $1.91 \mathrm{E}+03$ & $1.58 \mathrm{E}+02$ & $1.61 \mathrm{E}-05$ & $5.45 \mathrm{E}-09$ & $5.39 \mathrm{E}-09$ & $2.82 \mathrm{E}-04$ & $1.33 \mathrm{E}+00$ & $2.27 \mathrm{E}+02$ \\
\hline 3 & $6.77 \mathrm{E}+00$ & $6.93 \mathrm{E}+00$ & $6.90 \mathrm{E}+00$ & $7.10 \mathrm{E}+00$ & $7.30 \mathrm{E}+00$ & $7.70 \mathrm{E}+00$ & $7.63 \mathrm{E}+00$ & $7.58 \mathrm{E}+00$ & $7.68 \mathrm{E}+00$ \\
\hline 4 & $1.72 \mathrm{E}+00$ & $9.24 \mathrm{E}-01$ & $5.74 \mathrm{E}+01$ & $3.80 \mathrm{E}+02$ & $5.15 \mathrm{E}+02$ & $6.76 \mathrm{E}+02$ & $7.40 \mathrm{E}+02$ & $8.52 \mathrm{E}+02$ & $9.25 \mathrm{E}+02$ \\
\hline 5 & $1.25 \mathrm{E}+00$ & $1.21 \mathrm{E}+00$ & $1.28 \mathrm{E}+00$ & $1.30 \mathrm{E}+00$ & $1.30 \mathrm{E}+00$ & $1.31 \mathrm{E}+00$ & $1.28 \mathrm{E}+00$ & $1.27 \mathrm{E}+00$ & $1.34 \mathrm{E}+00$ \\
\hline 6 & $3.55 \mathrm{E}-02$ & $3.79 \mathrm{E}-02$ & $4.72 \mathrm{E}-02$ & $5.66 \mathrm{E}-02$ & $7.44 \mathrm{E}-02$ & $9.00 \mathrm{E}-02$ & $1.10 \mathrm{E}-01$ & $1.34 \mathrm{E}-01$ & $1.58 \mathrm{E}-01$ \\
\hline 7 & $1.65 \mathrm{E}-01$ & $7.64 \mathrm{E}-02$ & $5.17 \mathrm{E}-02$ & $5.10 \mathrm{E}-02$ & $5.30 \mathrm{E}-02$ & $6.74 \mathrm{E}-02$ & $7.87 \mathrm{E}-02$ & $7.99 \mathrm{E}-02$ & $9.35 \mathrm{E}-02$ \\
\hline 8 & $5.83 \mathrm{E}-01$ & $4.82 \mathrm{E}-01$ & $9.19 \mathrm{E}-01$ & $1.09 \mathrm{E}+00$ & $1.20 \mathrm{E}+00$ & $1.33 \mathrm{E}+00$ & $1.52 \mathrm{E}+00$ & $1.59 \mathrm{E}+00$ & $1.91 \mathrm{E}+00$ \\
\hline 9 & $1.22 \mathrm{E}+00$ & $1.06 \mathrm{E}+00$ & $1.32 \mathrm{E}+00$ & $1.84 \mathrm{E}+00$ & $2.22 \mathrm{E}+00$ & $2.42 \mathrm{E}+00$ & $2.55 \mathrm{E}+00$ & $2.64 \mathrm{E}+00$ & $2.71 \mathrm{E}+00$ \\
\hline 10 & $1.27 \mathrm{E}+03$ & $4.43 \mathrm{E}+02$ & $7.80 \mathrm{E}+01$ & $6.13 \mathrm{E}+01$ & $1.42 \mathrm{E}+02$ & $2.61 \mathrm{E}+02$ & $4.69 \mathrm{E}+02$ & $2.05 \mathrm{E}+03$ & $1.85 \mathrm{E}+04$ \\
\hline 11 & $2.96 \mathrm{E}+00$ & $3.13 \mathrm{E}+00$ & $3.89 \mathrm{E}+00$ & $4.79 \mathrm{E}+00$ & $5.43 \mathrm{E}+00$ & $6.44 \mathrm{E}+00$ & $8.02 \mathrm{E}+00$ & $1.08 \mathrm{E}+01$ & $1.16 \mathrm{E}+01$ \\
\hline 12 & $2.06 \mathrm{E}+01$ & $2.24 \mathrm{E}+01$ & $2.71 \mathrm{E}+01$ & $4.28 \mathrm{E}+01$ & $6.19 \mathrm{E}+01$ & $8.77 \mathrm{E}+01$ & $9.95 \mathrm{E}+01$ & $1.35 \mathrm{E}+02$ & $1.64 \mathrm{E}+02$ \\
\hline 13 & $2.11 \mathrm{E}+02$ & $2.10 \mathrm{E}+02$ & $2.10 \mathrm{E}+02$ & $2.10 \mathrm{E}+02$ & $2.10 \mathrm{E}+02$ & $2.11 \mathrm{E}+02$ & $2.12 \mathrm{E}+02$ & $2.14 \mathrm{E}+02$ & $2.23 \mathrm{E}+02$ \\
\hline 14 & $1.29 \mathrm{E}+02$ & $1.28 \mathrm{E}+02$ & $1.33 \mathrm{E}+02$ & $1.33 \mathrm{E}+02$ & $1.33 \mathrm{E}+02$ & $1.33 \mathrm{E}+02$ & $1.34 \mathrm{E}+02$ & $1.35 \mathrm{E}+02$ & $1.36 \mathrm{E}+02$ \\
\hline 15 & $2.98 \mathrm{E}+02$ & $2.96 \mathrm{E}+02$ & $3.12 \mathrm{E}+02$ & $3.27 \mathrm{E}+02$ & $3.44 \mathrm{E}+02$ & $3.40 \mathrm{E}+02$ & $3.50 \mathrm{E}+02$ & $3.55 \mathrm{E}+02$ & $3.59 \mathrm{E}+02$ \\
\hline
\end{tabular}

TABLE X.

RESULTS FOR POPULATION SIZE $100, \mathrm{CR}=0.8, \mathrm{~F}=0.4$

\begin{tabular}{|c|c|c|}
\hline $\boldsymbol{f}$ & Fitness & Percentage Change \\
\hline 1 & $1.50 \mathrm{E}+02$ & $>1000.00 \%$ \\
\hline 2 & $1.61 \mathrm{E}-05$ & $0.00 \%$ \\
\hline 3 & $7.10 \mathrm{E}+00$ & $-35.74 \%$ \\
\hline 4 & $3.80 \mathrm{E}+02$ & $-59.28 \%$ \\
\hline 5 & $1.30 \mathrm{E}+00$ & $-28.90 \%$ \\
\hline 6 & $5.66 \mathrm{E}-02$ & $-41.35 \%$ \\
\hline 7 & $5.10 \mathrm{E}-02$ & $-36.30 \%$ \\
\hline 8 & $1.09 \mathrm{E}+00$ & $-30.03 \%$ \\
\hline 9 & $1.84 \mathrm{E}+00$ & $-38.82 \%$ \\
\hline 10 & $6.13 \mathrm{E}+01$ & $288.70 \%$ \\
\hline 11 & $4.79 \mathrm{E}+00$ & $-19.41 \%$ \\
\hline 12 & $4.28 \mathrm{E}+01$ & $-12.38 \%$ \\
\hline 13 & $2.10 \mathrm{E}+02$ & $-33.41 \%$ \\
\hline 14 & $1.33 \mathrm{E}+02$ & $-32.78 \%$ \\
\hline 15 & $3.27 \mathrm{E}+02$ & $-32.97 \%$ \\
\hline & Overall & $-34.73 \%$ \\
\hline & Changes & \\
\hline
\end{tabular}


TABLE XI.

OVERALL RESULTS FOR POPULATION SIZE $150 \mathrm{CR}=0.8$

\begin{tabular}{|r|c|c|c|c|c|c|c|c|c|}
\hline $\begin{array}{r}\text { FR/ } \\
\boldsymbol{f}\end{array}$ & $\mathbf{0 . 1}$ & $\mathbf{0 . 2}$ & $\mathbf{0 . 3}$ & $\mathbf{0 . 4}$ & $\mathbf{0 . 5}$ & $\mathbf{0 . 6}$ & $\mathbf{0 . 7}$ & $\mathbf{0 . 8}$ & $\mathbf{0 . 9}$ \\
\hline 1 & $9.18 \mathrm{E}+03$ & $5.83 \mathrm{E}+03$ & $5.50 \mathrm{E}+03$ & $3.53 \mathrm{E}+00$ & $1.45 \mathrm{E}+03$ & $1.47 \mathrm{E}+05$ & $3.48 \mathrm{E}+06$ & $3.07 \mathrm{E}+07$ & $1.45 \mathrm{E}+08$ \\
\hline 2 & $3.36 \mathrm{E}+03$ & $9.47 \mathrm{E}+02$ & $1.22 \mathrm{E}+00$ & $5.27 \mathrm{E}-09$ & $5.13 \mathrm{E}-09$ & $1.39 \mathrm{E}-04$ & $3.74 \mathrm{E}-01$ & $8.96 \mathrm{E}+01$ & $2.84 \mathrm{E}+03$ \\
\hline 3 & $6.96 \mathrm{E}+00$ & $7.08 \mathrm{E}+00$ & $7.22 \mathrm{E}+00$ & $7.23 \mathrm{E}+00$ & $7.39 \mathrm{E}+00$ & $7.54 \mathrm{E}+00$ & $7.74 \mathrm{E}+00$ & $7.57 \mathrm{E}+00$ & $7.59 \mathrm{E}+00$ \\
\hline 4 & $7.49 \mathrm{E}-01$ & $1.35 \mathrm{E}+01$ & $1.59 \mathrm{E}+02$ & $4.65 \mathrm{E}+02$ & $5.74 \mathrm{E}+02$ & $6.98 \mathrm{E}+02$ & $7.78 \mathrm{E}+02$ & $8.79 \mathrm{E}+02$ & $9.44 \mathrm{E}+02$ \\
\hline 5 & $1.25 \mathrm{E}+00$ & $1.28 \mathrm{E}+00$ & $1.26 \mathrm{E}+00$ & $1.33 \mathrm{E}+00$ & $1.15 \mathrm{E}+00$ & $1.29 \mathrm{E}+00$ & $1.26 \mathrm{E}+00$ & $1.28 \mathrm{E}+00$ & $1.30 \mathrm{E}+00$ \\
\hline 6 & $3.82 \mathrm{E}-02$ & $3.96 \mathrm{E}-02$ & $4.69 \mathrm{E}-02$ & $5.81 \mathrm{E}-02$ & $8.09 \mathrm{E}-02$ & $9.67 \mathrm{E}-02$ & $1.24 \mathrm{E}-01$ & $1.39 \mathrm{E}-01$ & $1.68 \mathrm{E}-01$ \\
\hline 7 & $1.24 \mathrm{E}-01$ & $7.69 \mathrm{E}-02$ & $5.23 \mathrm{E}-02$ & $5.69 \mathrm{E}-02$ & $6.65 \mathrm{E}-02$ & $7.58 \mathrm{E}-02$ & $8.38 \mathrm{E}-02$ & $8.95 \mathrm{E}-02$ & $9.47 \mathrm{E}-02$ \\
\hline 8 & $5.44 \mathrm{E}-01$ & $5.76 \mathrm{E}-01$ & $9.95 \mathrm{E}-01$ & $1.17 \mathrm{E}+00$ & $1.22 \mathrm{E}+00$ & $1.38 \mathrm{E}+00$ & $1.59 \mathrm{E}+00$ & $1.71 \mathrm{E}+00$ & $1.88 \mathrm{E}+00$ \\
\hline 9 & $1.02 \mathrm{E}+00$ & $1.24 \mathrm{E}+00$ & $1.56 \mathrm{E}+00$ & $2.03 \mathrm{E}+00$ & $2.29 \mathrm{E}+00$ & $2.47 \mathrm{E}+00$ & $2.56 \mathrm{E}+00$ & $2.64 \mathrm{E}+00$ & $2.69 \mathrm{E}+00$ \\
\hline 10 & $8.30 \mathrm{E}+02$ & $1.82 \mathrm{E}+02$ & $6.14 \mathrm{E}+01$ & $1.15 \mathrm{E}+02$ & $1.95 \mathrm{E}+02$ & $3.63 \mathrm{E}+02$ & $1.20 \mathrm{E}+03$ & $8.92 \mathrm{E}+03$ & $4.78 \mathrm{E}+04$ \\
\hline 11 & $3.55 \mathrm{E}+00$ & $4.08 \mathrm{E}+00$ & $4.56 \mathrm{E}+00$ & $5.22 \mathrm{E}+00$ & $6.41 \mathrm{E}+00$ & $7.84 \mathrm{E}+00$ & $9.29 \mathrm{E}+00$ & $9.70 \mathrm{E}+00$ & $1.46 \mathrm{E}+01$ \\
\hline 12 & $2.20 \mathrm{E}+01$ & $2.61 \mathrm{E}+01$ & $3.35 \mathrm{E}+01$ & $4.92 \mathrm{E}+01$ & $7.32 \mathrm{E}+01$ & $8.79 \mathrm{E}+01$ & $1.30 \mathrm{E}+02$ & $1.39 \mathrm{E}+02$ & $1.63 \mathrm{E}+02$ \\
\hline 13 & $2.11 \mathrm{E}+02$ & $2.09 \mathrm{E}+02$ & $2.09 \mathrm{E}+02$ & $2.10 \mathrm{E}+02$ & $2.11 \mathrm{E}+02$ & $2.12 \mathrm{E}+02$ & $2.15 \mathrm{E}+02$ & $2.22 \mathrm{E}+02$ & $2.36 \mathrm{E}+02$ \\
\hline 14 & $1.28 \mathrm{E}+02$ & $1.29 \mathrm{E}+02$ & $1.33 \mathrm{E}+02$ & $1.33 \mathrm{E}+02$ & $1.33 \mathrm{E}+02$ & $1.34 \mathrm{E}+02$ & $1.34 \mathrm{E}+02$ & $1.35 \mathrm{E}+02$ & $1.37 \mathrm{E}+02$ \\
\hline 15 & $2.95 \mathrm{E}+02$ & $2.80 \mathrm{E}+02$ & $3.03 \mathrm{E}+02$ & $3.18 \mathrm{E}+02$ & $3.30 \mathrm{E}+02$ & $3.28 \mathrm{E}+02$ & $3.53 \mathrm{E}+02$ & $3.42 \mathrm{E}+02$ & $3.66 \mathrm{E}+02$ \\
\hline
\end{tabular}

TABLE XII.

RESUlTS FOR POPULATION SIZE $150, \mathrm{CR}=0.8, \mathrm{~F}=0.4$

\begin{tabular}{|c|c|c|}
\hline $\boldsymbol{f}$ & Fitness & Percentage Change \\
\hline 1 & $3.53 \mathrm{E}+00$ & $>1000.00 \%$ \\
\hline 2 & $5.27 \mathrm{E}-09$ & $0.00 \%$ \\
\hline 3 & $7.23 \mathrm{E}+00$ & $-34.58 \%$ \\
\hline 4 & $4.65 \mathrm{E}+02$ & $-50.18 \%$ \\
\hline 5 & $1.33 \mathrm{E}+00$ & $-27.30 \%$ \\
\hline 6 & $5.81 \mathrm{E}-02$ & $-39.77 \%$ \\
\hline 7 & $5.69 \mathrm{E}-02$ & $-28.93 \%$ \\
\hline 8 & $1.17 \mathrm{E}+00$ & $-25.11 \%$ \\
\hline 9 & $2.03 \mathrm{E}+00$ & $-32.37 \%$ \\
\hline 10 & $1.15 \mathrm{E}+02$ & $629.65 \%$ \\
\hline 11 & $5.22 \mathrm{E}+00$ & $-12.26 \%$ \\
\hline 12 & $4.92 \mathrm{E}+01$ & $0.80 \%$ \\
\hline 13 & $2.10 \mathrm{E}+02$ & $-33.35 \%$ \\
\hline 14 & $1.33 \mathrm{E}+02$ & $-32.78 \%$ \\
\hline 15 & $3.18 \mathrm{E}+02$ & $-34.85 \%$ \\
\hline & $\begin{array}{c}\text { Overall } \\
\text { Changes }\end{array}$ & $-35.18 \%$ \\
\hline & & \\
\hline
\end{tabular}


TABLE XIII.

OVERALL RESULTS FOR POPULATION SIZE 200,CR=0.9

\begin{tabular}{|r|c|c|c|c|c|c|c|c|c|}
\hline $\begin{array}{r}\text { FR/ } \\
\boldsymbol{f}\end{array}$ & $\mathbf{0 . 1}$ & $\mathbf{0 . 2}$ & $\mathbf{0 . 3}$ & $\mathbf{0 . 4}$ & $\mathbf{0 . 5}$ & $\mathbf{0 . 6}$ & $\mathbf{0 . 7}$ & $\mathbf{0 . 8}$ & $\mathbf{0 . 9}$ \\
\hline 1 & $5.83 \mathrm{E}+05$ & $6.80 \mathrm{E}+03$ & $1.68 \mathrm{E}+03$ & $4.01 \mathrm{E}-05$ & $8.49 \mathrm{E}-01$ & $1.37 \mathrm{E}+03$ & $3.09 \mathrm{E}+05$ & $1.05 \mathrm{E}+07$ & $1.02 \mathrm{E}+08$ \\
\hline 2 & $4.64 \mathrm{E}+03$ & $8.90 \mathrm{E}+02$ & $1.62 \mathrm{E}+00$ & $5.24 \mathrm{E}-09$ & $5.62 \mathrm{E}-09$ & $5.41 \mathrm{E}-09$ & $8.09 \mathrm{E}-07$ & $4.13 \mathrm{E}-02$ & $2.40 \mathrm{E}+01$ \\
\hline 3 & $7.21 \mathrm{E}+00$ & $7.23 \mathrm{E}+00$ & $7.22 \mathrm{E}+00$ & $7.38 \mathrm{E}+00$ & $7.44 \mathrm{E}+00$ & $7.65 \mathrm{E}+00$ & $7.70 \mathrm{E}+00$ & $7.59 \mathrm{E}+00$ & $7.88 \mathrm{E}+00$ \\
\hline 4 & $1.34 \mathrm{E}+00$ & $2.92 \mathrm{E}+01$ & $3.70 \mathrm{E}+02$ & $6.47 \mathrm{E}+02$ & $7.11 \mathrm{E}+02$ & $8.56 \mathrm{E}+02$ & $9.03 \mathrm{E}+02$ & $9.98 \mathrm{E}+02$ & $1.07 \mathrm{E}+03$ \\
\hline 5 & $1.32 \mathrm{E}+00$ & $1.28 \mathrm{E}+00$ & $1.28 \mathrm{E}+00$ & $1.35 \mathrm{E}+00$ & $1.26 \mathrm{E}+00$ & $1.23 \mathrm{E}+00$ & $1.29 \mathrm{E}+00$ & $1.33 \mathrm{E}+00$ & $1.25 \mathrm{E}+00$ \\
\hline 6 & $4.68 \mathrm{E}-02$ & $3.41 \mathrm{E}-02$ & $4.33 \mathrm{E}-02$ & $5.96 \mathrm{E}-02$ & $7.54 \mathrm{E}-02$ & $1.01 \mathrm{E}-01$ & $1.22 \mathrm{E}-01$ & $1.48 \mathrm{E}-01$ & $1.77 \mathrm{E}-01$ \\
\hline 7 & $1.30 \mathrm{E}-01$ & $6.57 \mathrm{E}-02$ & $5.17 \mathrm{E}-02$ & $5.94 \mathrm{E}-02$ & $6.52 \mathrm{E}-02$ & $7.53 \mathrm{E}-02$ & $8.55 \mathrm{E}-02$ & $9.20 \mathrm{E}-02$ & $1.02 \mathrm{E}-01$ \\
\hline 8 & $4.90 \mathrm{E}-01$ & $4.20 \mathrm{E}-01$ & $9.21 \mathrm{E}-01$ & $1.11 \mathrm{E}+00$ & $1.22 \mathrm{E}+00$ & $1.43 \mathrm{E}+00$ & $1.60 \mathrm{E}+00$ & $1.83 \mathrm{E}+00$ & $2.11 \mathrm{E}+00$ \\
\hline 9 & $1.28 \mathrm{E}+00$ & $1.16 \mathrm{E}+00$ & $1.56 \mathrm{E}+00$ & $1.99 \mathrm{E}+00$ & $2.26 \mathrm{E}+00$ & $2.41 \mathrm{E}+00$ & $2.55 \mathrm{E}+00$ & $2.65 \mathrm{E}+00$ & $2.70 \mathrm{E}+00$ \\
\hline 10 & $1.88 \mathrm{E}+03$ & $1.24 \mathrm{E}+02$ & $1.07 \mathrm{E}+01$ & $5.30 \mathrm{E}+01$ & $1.18 \mathrm{E}+02$ & $1.91 \mathrm{E}+02$ & $3.13 \mathrm{E}+02$ & $8.63 \mathrm{E}+02$ & $8.74 \mathrm{E}+03$ \\
\hline 11 & $3.58 \mathrm{E}+00$ & $3.73 \mathrm{E}+00$ & $4.25 \mathrm{E}+00$ & $4.79 \mathrm{E}+00$ & $5.82 \mathrm{E}+00$ & $6.71 \mathrm{E}+00$ & $8.05 \mathrm{E}+00$ & $1.08 \mathrm{E}+01$ & $1.14 \mathrm{E}+01$ \\
\hline 12 & $2.08 \mathrm{E}+01$ & $2.19 \mathrm{E}+01$ & $2.52 \mathrm{E}+01$ & $3.91 \mathrm{E}+01$ & $5.54 \mathrm{E}+01$ & $7.67 \mathrm{E}+01$ & $9.25 \mathrm{E}+01$ & $1.03 \mathrm{E}+02$ & $1.39 \mathrm{E}+02$ \\
\hline 13 & $2.12 \mathrm{E}+02$ & $2.10 \mathrm{E}+02$ & $2.10 \mathrm{E}+02$ & $2.10 \mathrm{E}+02$ & $2.10 \mathrm{E}+02$ & $2.12 \mathrm{E}+02$ & $2.24 \mathrm{E}+02$ & $2.38 \mathrm{E}+02$ & $2.67 \mathrm{E}+02$ \\
\hline 14 & $1.29 \mathrm{E}+02$ & $1.29 \mathrm{E}+02$ & $1.33 \mathrm{E}+02$ & $1.33 \mathrm{E}+02$ & $1.33 \mathrm{E}+02$ & $1.33 \mathrm{E}+02$ & $1.34 \mathrm{E}+02$ & $1.34 \mathrm{E}+02$ & $1.36 \mathrm{E}+02$ \\
\hline 15 & $2.94 \mathrm{E}+02$ & $2.97 \mathrm{E}+02$ & $3.08 \mathrm{E}+02$ & $3.11 \mathrm{E}+02$ & $3.30 \mathrm{E}+02$ & $3.32 \mathrm{E}+02$ & $3.42 \mathrm{E}+02$ & $3.41 \mathrm{E}+02$ & $3.55 \mathrm{E}+02$ \\
\hline
\end{tabular}

TABLE XIV.

RESULTS FOR POPULATION SIZE 200, $\mathrm{CR}=0.9, \mathrm{~F}=0.4$

\begin{tabular}{|c|c|c|}
\hline $\boldsymbol{f}$ & Fitness & Percentage Change \\
\hline 1 & $4.01 \mathrm{E}-05$ & $-53.81 \%$ \\
\hline 2 & $5.24 \mathrm{E}-09$ & $0.00 \%$ \\
\hline 3 & $7.38 \mathrm{E}+00$ & $-33.18 \%$ \\
\hline 4 & $6.47 \mathrm{E}+02$ & $-30.63 \%$ \\
\hline 5 & $1.35 \mathrm{E}+00$ & $-26.25 \%$ \\
\hline 6 & $5.96 \mathrm{E}-02$ & $-38.18 \%$ \\
\hline 7 & $5.94 \mathrm{E}-02$ & $-25.82 \%$ \\
\hline 8 & $1.11 \mathrm{E}+00$ & $-29.04 \%$ \\
\hline 9 & $1.99 \mathrm{E}+00$ & $-33.88 \%$ \\
\hline 10 & $5.30 \mathrm{E}+01$ & $236.25 \%$ \\
\hline 11 & $4.79 \mathrm{E}+00$ & $-19.49 \%$ \\
\hline 12 & $3.91 \mathrm{E}+01$ & $-19.97 \%$ \\
\hline 13 & $2.10 \mathrm{E}+02$ & $-33.45 \%$ \\
\hline 14 & $1.33 \mathrm{E}+02$ & $-32.82 \%$ \\
\hline 15 & $3.11 \mathrm{E}+02$ & $-36.25 \%$ \\
\hline & Overall & $-30.28 \%$ \\
\hline & Changes & \\
\hline
\end{tabular}


TABLE XV

OVERALL RESULTS FOR POPULATION SIZE $300, \mathrm{CR}=0.9$

\begin{tabular}{|r|c|c|c|c|c|c|c|c|c|}
\hline $\begin{array}{r}\text { FR/ } \\
\boldsymbol{f}\end{array}$ & $\mathbf{0 . 1}$ & $\mathbf{0 . 2}$ & $\mathbf{0 . 3}$ & $\mathbf{0 . 4}$ & $\mathbf{0 . 5}$ & $\mathbf{0 . 6}$ & $\mathbf{0 . 7}$ & $\mathbf{0 . 8}$ & $\mathbf{0 . 9}$ \\
\hline 1 & $5.83 \mathrm{E}+05$ & $6.80 \mathrm{E}+03$ & $1.68 \mathrm{E}+03$ & $4.01 \mathrm{E}-05$ & $8.49 \mathrm{E}-01$ & $1.37 \mathrm{E}+03$ & $3.09 \mathrm{E}+05$ & $1.05 \mathrm{E}+07$ & $1.02 \mathrm{E}+08$ \\
\hline 2 & $4.64 \mathrm{E}+03$ & $8.90 \mathrm{E}+02$ & $1.62 \mathrm{E}+00$ & $5.24 \mathrm{E}-09$ & $5.62 \mathrm{E}-09$ & $5.41 \mathrm{E}-09$ & $8.09 \mathrm{E}-07$ & $4.13 \mathrm{E}-02$ & $2.40 \mathrm{E}+01$ \\
\hline 3 & $7.21 \mathrm{E}+00$ & $7.23 \mathrm{E}+00$ & $7.22 \mathrm{E}+00$ & $7.38 \mathrm{E}+00$ & $7.44 \mathrm{E}+00$ & $7.65 \mathrm{E}+00$ & $7.70 \mathrm{E}+00$ & $7.59 \mathrm{E}+00$ & $7.88 \mathrm{E}+00$ \\
\hline 4 & $1.34 \mathrm{E}+00$ & $2.92 \mathrm{E}+01$ & $3.70 \mathrm{E}+02$ & $6.47 \mathrm{E}+02$ & $7.11 \mathrm{E}+02$ & $8.56 \mathrm{E}+02$ & $9.03 \mathrm{E}+02$ & $9.98 \mathrm{E}+02$ & $1.07 \mathrm{E}+03$ \\
\hline 5 & $1.32 \mathrm{E}+00$ & $1.28 \mathrm{E}+00$ & $1.28 \mathrm{E}+00$ & $1.35 \mathrm{E}+00$ & $1.26 \mathrm{E}+00$ & $1.23 \mathrm{E}+00$ & $1.29 \mathrm{E}+00$ & $1.33 \mathrm{E}+00$ & $1.25 \mathrm{E}+00$ \\
\hline 6 & $4.68 \mathrm{E}-02$ & $3.41 \mathrm{E}-02$ & $4.33 \mathrm{E}-02$ & $5.96 \mathrm{E}-02$ & $7.54 \mathrm{E}-02$ & $1.01 \mathrm{E}-01$ & $1.22 \mathrm{E}-01$ & $1.48 \mathrm{E}-01$ & $1.77 \mathrm{E}-01$ \\
\hline 7 & $1.30 \mathrm{E}-01$ & $6.57 \mathrm{E}-02$ & $5.17 \mathrm{E}-02$ & $5.94 \mathrm{E}-02$ & $6.52 \mathrm{E}-02$ & $7.53 \mathrm{E}-02$ & $8.55 \mathrm{E}-02$ & $9.20 \mathrm{E}-02$ & $1.02 \mathrm{E}-01$ \\
\hline 8 & $4.90 \mathrm{E}-01$ & $4.20 \mathrm{E}-01$ & $9.21 \mathrm{E}-01$ & $1.11 \mathrm{E}+00$ & $1.22 \mathrm{E}+00$ & $1.43 \mathrm{E}+00$ & $1.60 \mathrm{E}+00$ & $1.83 \mathrm{E}+00$ & $2.11 \mathrm{E}+00$ \\
\hline 9 & $1.28 \mathrm{E}+00$ & $1.16 \mathrm{E}+00$ & $1.56 \mathrm{E}+00$ & $1.99 \mathrm{E}+00$ & $2.26 \mathrm{E}+00$ & $2.41 \mathrm{E}+00$ & $2.55 \mathrm{E}+00$ & $2.65 \mathrm{E}+00$ & $2.70 \mathrm{E}+00$ \\
\hline 10 & $1.88 \mathrm{E}+03$ & $1.24 \mathrm{E}+02$ & $1.07 \mathrm{E}+01$ & $5.30 \mathrm{E}+01$ & $1.18 \mathrm{E}+02$ & $1.91 \mathrm{E}+02$ & $3.13 \mathrm{E}+02$ & $8.63 \mathrm{E}+02$ & $8.74 \mathrm{E}+03$ \\
\hline 11 & $3.58 \mathrm{E}+00$ & $3.73 \mathrm{E}+00$ & $4.25 \mathrm{E}+00$ & $4.79 \mathrm{E}+00$ & $5.82 \mathrm{E}+00$ & $6.71 \mathrm{E}+00$ & $8.05 \mathrm{E}+00$ & $1.08 \mathrm{E}+01$ & $1.14 \mathrm{E}+01$ \\
\hline 12 & $2.08 \mathrm{E}+01$ & $2.19 \mathrm{E}+01$ & $2.52 \mathrm{E}+01$ & $3.91 \mathrm{E}+01$ & $5.54 \mathrm{E}+01$ & $7.67 \mathrm{E}+01$ & $9.25 \mathrm{E}+01$ & $1.03 \mathrm{E}+02$ & $1.39 \mathrm{E}+02$ \\
\hline 13 & $2.12 \mathrm{E}+02$ & $2.10 \mathrm{E}+02$ & $2.10 \mathrm{E}+02$ & $2.10 \mathrm{E}+02$ & $2.10 \mathrm{E}+02$ & $2.12 \mathrm{E}+02$ & $2.24 \mathrm{E}+02$ & $2.38 \mathrm{E}+02$ & $2.67 \mathrm{E}+02$ \\
\hline 14 & $1.29 \mathrm{E}+02$ & $1.29 \mathrm{E}+02$ & $1.33 \mathrm{E}+02$ & $1.33 \mathrm{E}+02$ & $1.33 \mathrm{E}+02$ & $1.33 \mathrm{E}+02$ & $1.34 \mathrm{E}+02$ & $1.34 \mathrm{E}+02$ & $1.36 \mathrm{E}+02$ \\
\hline 15 & $2.94 \mathrm{E}+02$ & $2.97 \mathrm{E}+02$ & $3.08 \mathrm{E}+02$ & $3.11 \mathrm{E}+02$ & $3.30 \mathrm{E}+02$ & $3.32 \mathrm{E}+02$ & $3.42 \mathrm{E}+02$ & $3.41 \mathrm{E}+02$ & $3.55 \mathrm{E}+02$ \\
\hline
\end{tabular}

TABLE XVI.

RESULTS FOR POPULATION SIZE $300, \mathrm{CR}=0.9, \mathrm{~F}=0.3$

\begin{tabular}{|c|c|c|}
\hline $\boldsymbol{f}$ & Fitness & Percentage Change \\
\hline 1 & $2.35 \mathrm{E}+02$ & $>1000.00 \%$ \\
\hline 2 & $3.25 \mathrm{E}-03$ & $0.32 \%$ \\
\hline 3 & $7.54 \mathrm{E}+00$ & $-31.79 \%$ \\
\hline 4 & $4.71 \mathrm{E}+02$ & $-49.44 \%$ \\
\hline 5 & $1.31 \mathrm{E}+00$ & $-28.66 \%$ \\
\hline 6 & $4.81 \mathrm{E}-02$ & $-50.14 \%$ \\
\hline 7 & $6.22 \mathrm{E}-02$ & $-22.36 \%$ \\
\hline 8 & $1.02 \mathrm{E}+00$ & $-34.93 \%$ \\
\hline 9 & $1.78 \mathrm{E}+00$ & $-40.89 \%$ \\
\hline 10 & $1.95 \mathrm{E}+01$ & $23.77 \%$ \\
\hline 11 & $4.92 \mathrm{E}+00$ & $-17.25 \%$ \\
\hline 12 & $3.04 \mathrm{E}+01$ & $-37.69 \%$ \\
\hline 13 & $2.09 \mathrm{E}+02$ & $-33.73 \%$ \\
\hline 14 & $1.33 \mathrm{E}+02$ & $-32.77 \%$ \\
\hline 15 & $3.06 \mathrm{E}+02$ & $-37.19 \%$ \\
\hline & Overall & $-29.69 \%$ \\
\hline & & \\
\hline
\end{tabular}


TABLE XVII.

OVERALL RESULTS FOR POPULATION SIZE 500, CR=0.9

\begin{tabular}{|c|c|c|c|c|c|c|c|c|c|}
\hline $\begin{array}{c}\text { FR/ } \\
f\end{array}$ & 0.1 & 0.2 & 0.3 & 0.4 & 0.5 & 0.6 & 0.7 & 0.8 & 0.9 \\
\hline 1 & $7.01 \mathrm{E}+03$ & $5.26 \mathrm{E}+03$ & $7.16 \mathrm{E}+01$ & $4.91 \mathrm{E}+03$ & $2.39 \mathrm{E}+05$ & $4.56 \mathrm{E}+06$ & $3.86 \mathrm{E}+07$ & $1.85 \mathrm{E}+08$ & $5.05 \mathrm{E}+08$ \\
\hline 2 & $1.84 \mathrm{E}+03$ & $7.70 \mathrm{E}+01$ & $5.40 \mathrm{E}-09$ & 5.34E-09 & $3.31 \mathrm{E}-06$ & $1.39 \mathrm{E}-02$ & $5.67 \mathrm{E}+00$ & $4.16 \mathrm{E}+02$ & $6.14 \mathrm{E}+03$ \\
\hline 3 & $7.49 \mathrm{E}+00$ & $7.59 \mathrm{E}+00$ & $7.43 \mathrm{E}+00$ & $7.71 \mathrm{E}+00$ & $7.53 \mathrm{E}+00$ & $7.47 \mathrm{E}+00$ & $7.43 \mathrm{E}+00$ & $7.40 \mathrm{E}+00$ & $7.77 \mathrm{E}+00$ \\
\hline 4 & $2.43 \mathrm{E}+01$ & $1.42 \mathrm{E}+02$ & $5.47 \mathrm{E}+02$ & $6.82 \mathrm{E}+02$ & $7.36 \mathrm{E}+02$ & $8.84 \mathrm{E}+02$ & $9.30 \mathrm{E}+02$ & $9.97 \mathrm{E}+02$ & $1.05 \mathrm{E}+03$ \\
\hline 5 & $1.35 \mathrm{E}+00$ & $1.32 \mathrm{E}+00$ & $1.27 \mathrm{E}+00$ & $1.21 \mathrm{E}+00$ & $1.30 \mathrm{E}+00$ & $1.26 \mathrm{E}+00$ & $1.22 \mathrm{E}+00$ & $1.28 \mathrm{E}+00$ & $1.26 \mathrm{E}+00$ \\
\hline 6 & $3.42 \mathrm{E}-02$ & $3.71 \mathrm{E}-02$ & $5.22 \mathrm{E}-02$ & 7.25E-02 & $9.02 \mathrm{E}-02$ & $1.14 \mathrm{E}-01$ & $1.43 \mathrm{E}-01$ & $1.82 \mathrm{E}-01$ & $2.21 \mathrm{E}-01$ \\
\hline 7 & $9.76 \mathrm{E}-02$ & $6.65 \mathrm{E}-02$ & 7.63E-02 & 7.97E-02 & $8.57 \mathrm{E}-02$ & $8.90 \mathrm{E}-02$ & $9.25 \mathrm{E}-02$ & $9.97 \mathrm{E}-02$ & $1.12 \mathrm{E}-01$ \\
\hline 8 & $3.59 \mathrm{E}-01$ & 8.83E-01 & $1.14 \mathrm{E}+00$ & $1.19 \mathrm{E}+00$ & $1.41 \mathrm{E}+00$ & $1.51 \mathrm{E}+00$ & $1.75 \mathrm{E}+00$ & $2.01 \mathrm{E}+00$ & $2.56 \mathrm{E}+00$ \\
\hline 9 & $1.18 \mathrm{E}+00$ & $1.59 \mathrm{E}+00$ & $1.94 \mathrm{E}+00$ & $2.18 \mathrm{E}+00$ & $2.34 \mathrm{E}+00$ & $2.47 \mathrm{E}+00$ & $2.53 \mathrm{E}+00$ & $2.58 \mathrm{E}+00$ & $2.67 \mathrm{E}+00$ \\
\hline 10 & $2.71 \mathrm{E}+02$ & 4.18E+01 & $6.48 \mathrm{E}+01$ & $1.49 \mathrm{E}+02$ & $2.37 \mathrm{E}+02$ & $5.12 \mathrm{E}+02$ & $2.30 \mathrm{E}+03$ & $1.51 \mathrm{E}+04$ & $7.62 \mathrm{E}+04$ \\
\hline 11 & $5.57 \mathrm{E}+00$ & $6.04 \mathrm{E}+00$ & $6.30 \mathrm{E}+00$ & $6.96 \mathrm{E}+00$ & $8.20 \mathrm{E}+00$ & $8.92 \mathrm{E}+00$ & $1.05 \mathrm{E}+01$ & $1.18 \mathrm{E}+01$ & $1.21 \mathrm{E}+01$ \\
\hline 12 & $2.38 \mathrm{E}+01$ & $2.98 \mathrm{E}+01$ & $4.07 \mathrm{E}+01$ & $5.13 \mathrm{E}+01$ & $6.53 \mathrm{E}+01$ & $8.25 \mathrm{E}+01$ & $1.07 \mathrm{E}+02$ & $1.30 \mathrm{E}+02$ & $1.47 \mathrm{E}+02$ \\
\hline 13 & $2.10 \mathrm{E}+02$ & $2.09 \mathrm{E}+02$ & $2.10 \mathrm{E}+02$ & $2.11 \mathrm{E}+02$ & $2.13 \mathrm{E}+02$ & $2.21 \mathrm{E}+02$ & $2.47 \mathrm{E}+02$ & $2.66 \mathrm{E}+02$ & $2.74 \mathrm{E}+02$ \\
\hline 14 & $1.27 \mathrm{E}+02$ & $1.32 \mathrm{E}+02$ & $1.33 \mathrm{E}+02$ & $1.33 \mathrm{E}+02$ & $1.34 \mathrm{E}+02$ & $1.34 \mathrm{E}+02$ & $1.34 \mathrm{E}+02$ & $1.36 \mathrm{E}+02$ & $1.37 \mathrm{E}+02$ \\
\hline 15 & $3.03 \mathrm{E}+02$ & $2.95 \mathrm{E}+02$ & $2.88 \mathrm{E}+02$ & $3.02 \mathrm{E}+02$ & $3.06 \mathrm{E}+02$ & $3.14 \mathrm{E}+02$ & $3.17 \mathrm{E}+02$ & $3.24 \mathrm{E}+02$ & $3.24 \mathrm{E}+02$ \\
\hline
\end{tabular}

